\title{
Can endovascular repair be the first-line treatment option in abdominal aortic aneurysms during COVID-19 pandemic?
}

\author{
Kaptanıderya Tayfur (D), Melih Ürkmez (D) \\ Department of Cardiovascular Surgery, Ordu University, Training and Research Hospital, Ordu, Turkey
}

\begin{abstract}
Objectives: This study aims to investigate the effectiveness of endovascular aneurysm repair (EVAR) of abdominal aortic aneurysms (AAAs) during the novel coronavirus-2019 (COVID-19) pandemic.

Patients and methods: A total of 22 patients ( 17 males, 5 females; mean age: $76.7 \pm 7.1$ years; range, 66 to 90 years) who underwent EVAR for AAAs in our center between March 2020 and December 2020 were retrospectively analyzed. All patients underwent reverse transcriptasepolymerase chain reaction (RT-PCR) for severe acute respiratory syndrome-coronavirus 2 (SARS-CoV-2) infection. All EVAR procedures were performed under spinal anesthesia in the elective setting in the angiography laboratory.

Results: The RT-PCR test result was positive in six patients with asymptomatic infection. The mean AAA diameter was $74.7 \pm 5.5$ (range, 68.50 to 85.60 ) $\mathrm{mm}$. Stent-graft implantation was successfully performed in all patients. There was no significant relationship between the mean age and examined variables ( $p>0.050)$. The mean AAA diameter was $83.95 \mathrm{~mm}$ in the COVID-19-positive patients and $71.50 \mathrm{~mm}$ in the COVID-19-negative patients, indicating a significant difference ( $\mathrm{p}=0.001$ ). No mortality, stent migration or thrombotic events were observed during the first 30 days of follow-up.

Conclusion: The operation can be delayed in patients requiring open surgical repair or in those with small AAAs. Based on our findings, the EVAR can be used as the first-line treatment option during the pandemic, as it does not require intubation and requires a low number of staff and is cost-effective with minimum necessity for intensive care unit stay and less use of healthcare resources.
\end{abstract}

Keywords: Aneurysm, COVID-19, endovascular.

Pneumonia of unknown cause was first identified in Wuhan, Hubei province of China in December 2019 and novel coronavirus-2019 (COVID-19), caused by severe acute respiratory syndrome-coronavirus 2 (SARS-CoV-2), was emerged and rapidly spread to the world, resulting in a devastating pandemic. ${ }^{[1]}$ The first case of COVID-19 was identified in Turkey on March $11^{\text {th }}$, 2020 and affected many aspects of life with an increasing risk for millions of people. ${ }^{[1]}$

The COVID-19 pandemic has dramatically affected healthcare services in many ways worldwide, and surgical workforce are the most severely affected group. The working practices and treatment methods have been considerably changed for surgeons, forcing them to adopt new working practices. In particular, the pandemic has increased the need for intensive care with the unmet additional demand for intensive care unit (ICU) and hospital beds and staff, leading to a substantial burden on the healthcare resources across the world. In many settings, the ICU beds have been occupied by COVID-19 patients and treatment options has decreased in those suffering from diseases other than COVID-19. ${ }^{[2]}$

A recent study investigating vascular surgeries worldwide during the pandemic demonstrated that the number of elective surgeries significantly decreased

Received: February 09, 2021 Accepted: March 05, 2021 Published online: March 24, 2021

Correspondence: Kaptanıderya Tayfur, MD. Ordu Üniversitesi Eğitim ve Araştırma Hastanesi Kalp ve Damar Cerrahisi Kliniği, 52200 Altınordu, Ordu, Türkiye. e-mail: drkdtayfur61@hotmail.com 
in many centers. ${ }^{[3]}$ In addition, the American College of Surgeons issued guidelines for elective surgery and recommended that surgery should be postponed for abdominal aortic aneurysms (AAAs) larger than $6.5 \mathrm{~cm}$ (Tier 2b) and smaller than $6.5 \mathrm{~cm}$ (Tier 1), if possible. ${ }^{[4]}$ However, delayed treatment in specifically vascular diseases which are of vital importance and in whom timely treatment is critical is associated with impaired quality of life and increased morbidity and mortality rates. ${ }^{[5]}$

The treatment of AAAs in patients with and without COVID-19 during the pandemic is still controversial and no data are available, except for a few case reports in the literature. In the present study, therefore, we aimed to investigate the effectiveness of endovascular aneurysm repair (EVAR) of AAAs during the COVID-19 pandemic to provide a contribution to the body of knowledge in the literature.

\section{PATIENTS AND METHODS}

This single-center, retrospective study was conducted at Ordu Training and Research Hospital, Department of Cardiovascular Surgery between March 2020 and December 2020. A total of 22 patients (17 males, 5 females; mean age: $76.7 \pm 7.1$ years; range, 66 to 90 years) who underwent EVAR for AAAs in our center were included. Aneurysms less than $6.5 \mathrm{~mm}$ in diameter were excluded. Indications of EVAR for AAAs were compatible with the American College of Surgeons guidelines and AAAs of $<6.5 \mathrm{~cm}$ were left untreated (Table 1). Prior to surgery, all patients were informed about the possible risks and benefits of the procedure and a written informed consent was obtained. The study protocol was approved by the Ordu University, Faculty of Medicine, Ethics Committee (No: 2021/30). The study was conducted in accordance with the principles of the Declaration of Helsinki.
Data including demographic and clinical characteristics of the patients, imaging study findings, pre-, intra-, and postoperative data were recorded. All patients underwent reverse transcriptase-polymerase chain reaction (RT-PCR) for SARS-CoV-2 infection. The diameters of AAAs were measured using the computed tomography angiography (CTA) and stent-grafts to be deployed were selected.

All EVAR procedures were performed under spinal anesthesia in the elective setting in the angiography lab with a minimum number of staff (one surgeon, one nurse, and one CTA technician). All healthcare workers complied with the donning/doffing procedures of personal protective equipment including such as face mask, gloves, goggles, and face shields). Before the procedure, heparin at a standard dose $(5,000 \mathrm{U})$ was administered to the COVID-19-negative patients, while COVID-19-positive patients received heparin with an activated clotting time (ACT) of $>300 \mathrm{sec}$ to prevent thrombotic events. In all patients, the Endurant $^{\mathrm{TM}}$ (Medtronic Vascular, CA, USA) stentgraft was used. Balloon dilatation was applied in the patients with type 1 endoleak following the procedure, as confirmed by aortography or an aortic cuff was placed to prevent endoleaks intraoperatively. All patients received anticoagulants after the procedure. The COVID-19-positive patients were given additional favipiravir and followed in the COVID-19 ward.

\section{Statistical analysis}

Statistical analysis was performed using the IBM SPSS version 23.0 software (IBM Corp., Armonk, NY, USA). Descriptive data were expressed in mean \pm standard deviation (SD), median (min-max) or number and frequency. The normality assumption was checked using the Shapiro-Wilk test. The independent samples t-test was used to compare normally distributed

Table 1. The American College of Surgeons guidelines for elective surgery during COVID-19
pandemic
\begin{tabular}{ccc} 
Category & Condition & Tier class \\
\hline AAA & Ruptured or symptomatic TAAA or AAA & 3 do not postpone \\
AAA & Aneurysm associated infection or prosthetic graft infection & 3 do not postpone \\
AAA & AAA $>6.5 \mathrm{~cm}$ & $2 b$ postpone if possible \\
AAA & TAAA $>6.5 \mathrm{~cm}$ & $2 b$ postpone if possible \\
AAA & AAA $<6.5 \mathrm{~cm}$ & 1 postpone
\end{tabular}

AAA: Abdominal aortic aneurysm; TAAA: Torakoabdominal aortic aneurysm. 
quantitative variables between the groups, while the Mann-Whitney $U$ test was used to compare nonnormally distributed variables. A $p$ value of $<0.05$ was considered statistically significant.

\section{RESULTS}

All patients were symptomatic and low back pain and persistent abdominal pain were the most common symptoms. In one patient, additional dissection extending toward the right iliac artery was detected (Figure 1a-c). Another patient had a coexisting left common iliac artery aneurysm of $7 \mathrm{~cm}$ in size (Figure 2a, b). The mean length of hospital stay was $1.77 \pm 0.75$ (range, 1 to 3 ) days. Quantitative descriptive variables are summarized in Table 2 .

The RT-PCR test result was positive in six patients with asymptomatic infection. Of the patients, all had hypertension $(\mathrm{n}=22)$, followed by chronic obstructive pulmonary disease $(n=13)$, diabetes $(n=8)$, and heart failure $(n=4)$. Eight patients had previous coronary artery bypass grafting, while seven patients were smokers. The categorical variables are shown in Table 3.

The mean AAA diameter was $74.7 \pm 5.5$ (range, 68.50 to 85.60$) \mathrm{mm}$. The mean AAA diameter was $83.95 \mathrm{~mm}$ in the COVID-19-positive patients and $71.50 \mathrm{~mm}$ in the COVID-19-negative patients, indicating a significant difference $(\mathrm{p}=0.001)$. The mean AAA diameter was $74.63 \pm 5.50 \mathrm{~mm}$ in male patients and $75.0 \pm 6.3 \mathrm{~mm}$ in female patients, indicating no significant difference between the sexes $(p=0.820)$. The comparison of the variables according to the sex is presented in Table 4.

Stent-graft implantation was successfully performed in all patients (Figure 3a-c). Following the procedure, balloon dilatation was applied to two of three patients with type 1 endoleak, as confirmed by aortography and an aortic cuff was placed in one patient to prevent endoleaks intraoperatively. No intra- or postoperative complications were observed and none of the patients required blood transfusion.

None of the patients needed ICU stay and were transferred to the ward in the postoperative period. The COVID-19-positive patients were given additional favipiravir and followed in the COVID-19 ward. The mean age was significantly higher in the patients with COVID-19 than those without $(82.7 \pm 5.5$ years vs. $74.5 \pm 6.5$ years, respectively; $\mathrm{p}=0.013$ ). In addition, the mean AAA diameter was significantly higher in the patients with COVID-19 than those without
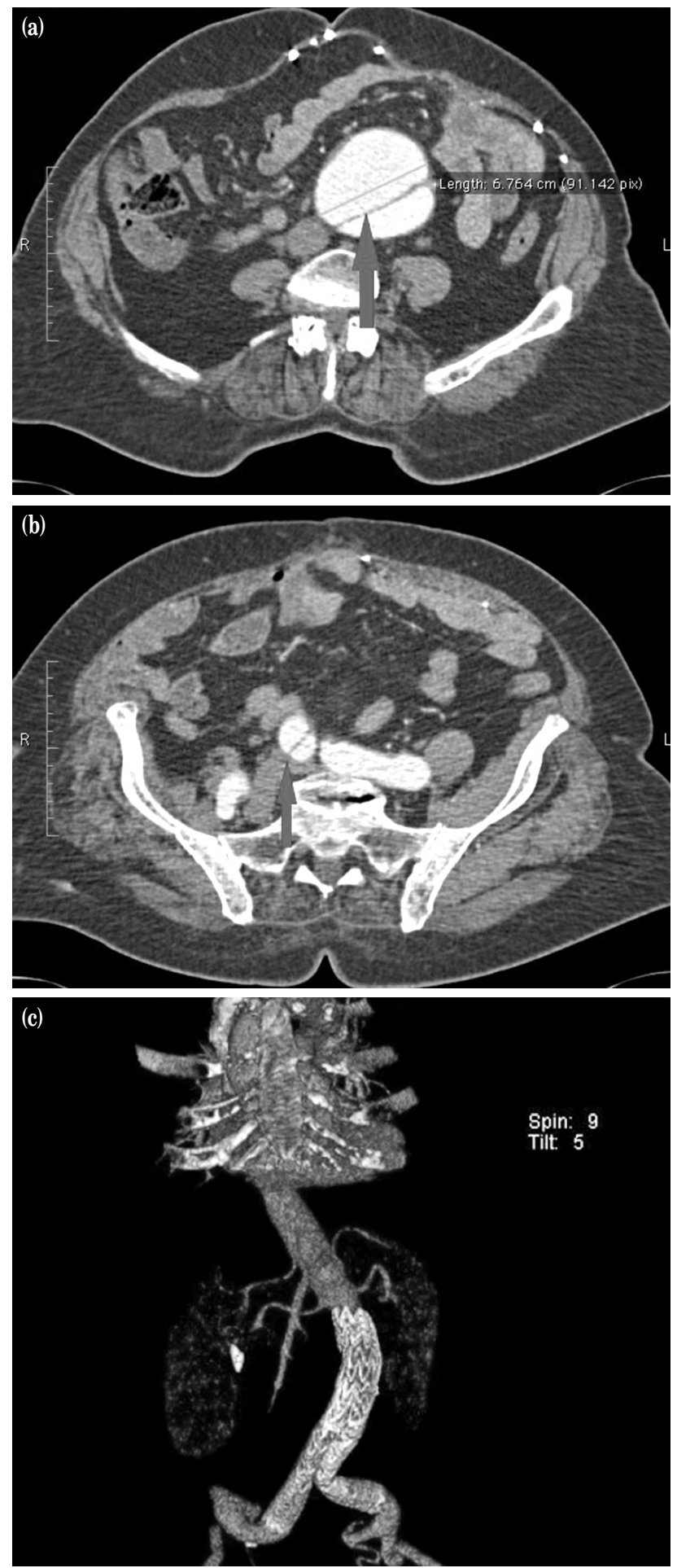

Figure 1. (a) A preoperative CT image showing AAA and coexisting dissection. (b) Right iliac artery dissection. (c) Postoperative CT image of the patient.

CT: Computed tomography; AAA: Abdominal aortic aneurysms.

(82.0 $\pm 5.1 \mathrm{~mm}$ vs. $72.0 \pm 2.3 \mathrm{~mm}$, respectively; $\mathrm{p}=0.001)$. There was also a significant difference in the mean length of hospital stay between the patients with and 

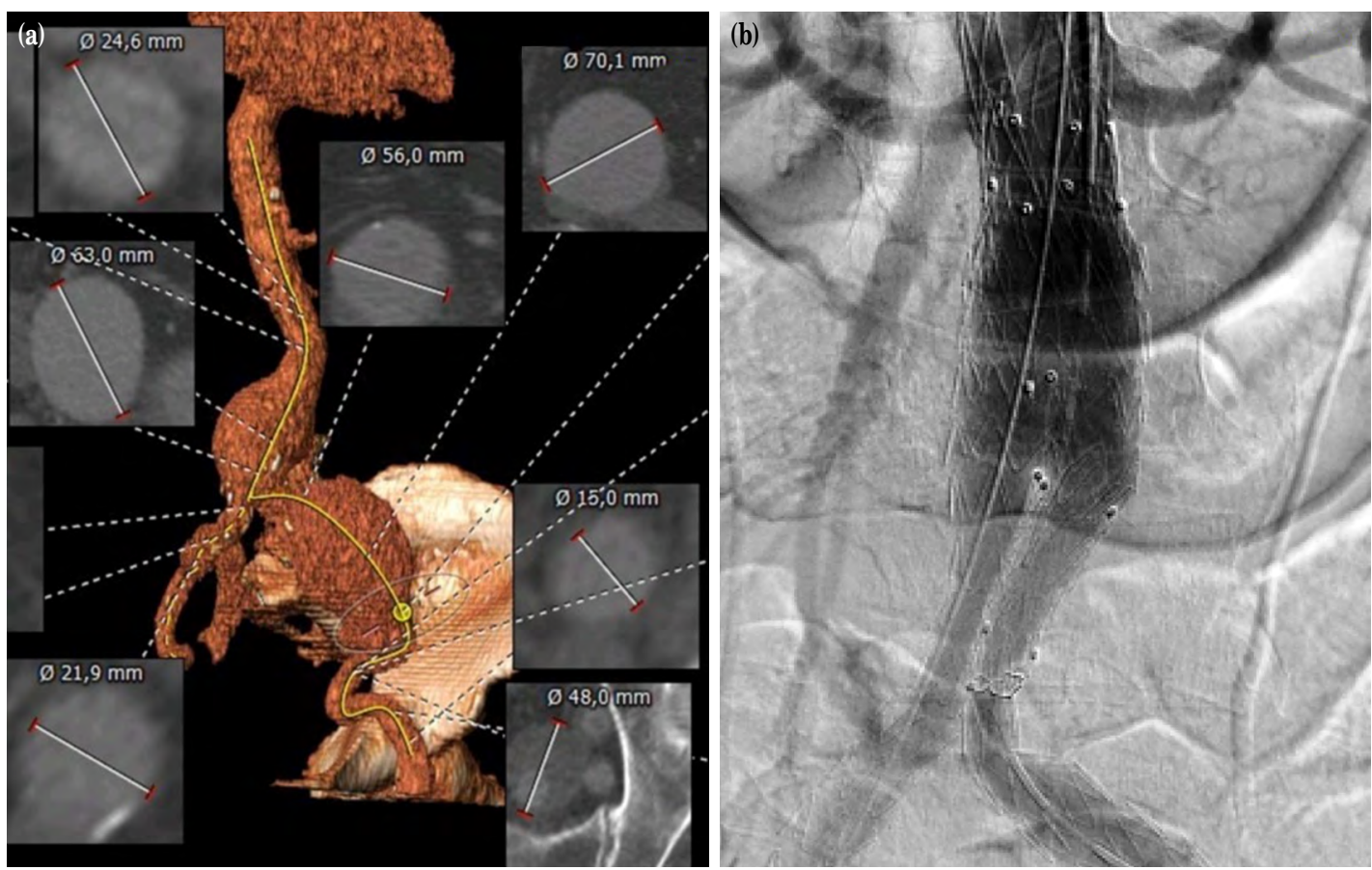

Figure 2. (a) A preoperative CT image showing AAA and left main iliac artery aneurysm. (b) Postoperative aortography showing of the patient.

CT: Computed tomography; AAA: Abdominal aortic aneurysms.

\section{Table 2. Quantitative descriptive variables}

\begin{tabular}{lccc}
\hline Variables & Mean \pm SD & Median & Min-Max \\
\hline Age (year) & $76.7 \pm 7.1$ & 77.00 & $60.00-90.00$ \\
Abdominal aortic diameter $(\mathrm{mm})$ & $74.7 \pm 5.5$ & 73.60 & $68.50-85.60$ \\
Aortic neck diameter $(\mathrm{mm})$ & $26.6 \pm 3.6$ & 27.00 & $16.50-31.20$ \\
Aortic neck length $(\mathrm{mm})$ & $18.5 \pm 2.8$ & 17.50 & $14.00-24.00$ \\
Right iliac diameter $(\mathrm{mm})$ & $24.5 \pm 10.9$ & 21.50 & $14.60-64.50$ \\
Left iliac diameter $(\mathrm{mm})$ & $20.5 \pm 4.3$ & 18.65 & $14.30-31.50$ \\
Procedural duration $(\mathrm{mm})$ & $52.0 \pm 7.3$ & 51.00 & $40.00-65.00$ \\
Length of hospital stay (days) & $1.8 \pm 0.8$ & 2.00 & $1.00-3.00$ \\
\hline
\end{tabular}

SD: Standard deviation; Min: Minimum; Max: Maximum.

\begin{tabular}{lcc} 
Table 3. Distribution of categorical variables & & \\
\hline Variables & $\mathrm{n}$ & $\%$ \\
\hline Sex & & \\
$\quad$ Male & 17 & 77.3 \\
Female & 5 & 22.7 \\
COVID-19 & & \\
$(-)$ & 16 & 72.7 \\
$(+)$ & 6 & 27.3 \\
Comorbidity* & & \\
Diabetes & 8 & 36.4 \\
Chronic obstructive pulmonary disease & 13 & 59.1 \\
Heart failure & 4 & 18.2 \\
Smoking & 7 & 31.8 \\
Previous coronary artery bypass grafting & 8 & 36.4 \\
Hypertension & 22 & 100.0 \\
\hline
\end{tabular}

COVID-19: novel coronavirus-2019; * Multiple responses.

Table 4. The comparison of the variables according to the sex

\begin{tabular}{|c|c|c|c|c|c|c|c|c|}
\hline & \multicolumn{3}{|c|}{ Male } & \multicolumn{3}{|c|}{ Female } & \multirow[b]{2}{*}{ Test statistics } & \multirow[b]{2}{*}{$p$} \\
\hline & Mean \pm SD & Median & Min-Max & Mean \pm SD & Median & Min-Max & & \\
\hline Age (year) & $76.0 \pm 6.6$ & 77.00 & $60.00-87.00$ & $79.2 \pm 9.0$ & 80.00 & $66.00-90.00$ & $-0.878 \dagger$ & 0.390 \\
\hline Abdominal aortic diameter (mm) & $74.6 \pm 5.5$ & 73.60 & $69.10-85.60$ & $75.0 \pm 6.3$ & 74.20 & $68.50-85.30$ & 46.000 & 0.820 \\
\hline Aortic neck diameter $(\mathrm{mm})$ & $26.5 \pm 3.8$ & 26.80 & $16.50-31.20$ & $26.8 \pm 3.2$ & 27.20 & $21.70-29.50$ & $-0.178 \dagger$ & 0.860 \\
\hline Aortic neck length (mm) & $18.5 \pm 3.0$ & 18.00 & $14.00-24.00$ & $18.2 \pm 2.2$ & 17.00 & $16.00-21.00$ & $0.228 \dagger$ & 0.822 \\
\hline Right iliac diameter (mm) & $24.6 \pm 12.2$ & 20.50 & $14.60-64.50$ & $24.2 \pm 5.0$ & 24.40 & $19.50-32.30$ & 56.000 & 0.319 \\
\hline Left iliac diameter (mm) & $20.0 \pm 3.8$ & 18.60 & $14.30-26.30$ & $22.0 \pm 6.1$ & 22.40 & $15.30-31.50$ & $-0.91 \dagger$ & 0.374 \\
\hline
\end{tabular}

SD: Standard deviation; Min: Minimum; Max: Maximum; $†$ Independent samples t-test; $¥$ Mann-Whitney U test. 

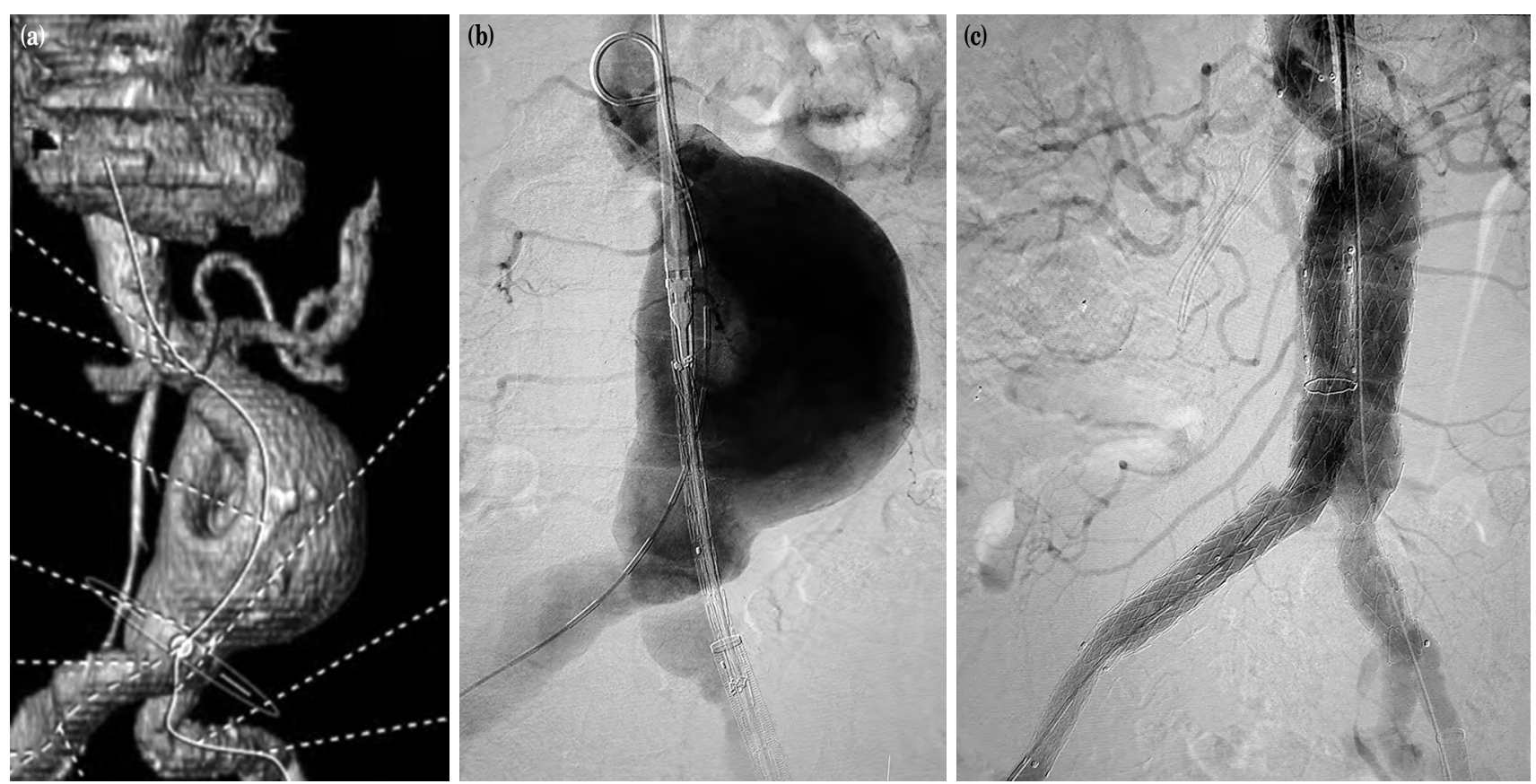

Figure 3. (a) A preoperative CT image showing a giant AAA with severe aortic neck angulation. (b) Preoperative aortography showing of the patient. (c) Postoperative aortography showing of the patient.

CT: Computed tomography; AAA: Abdominal aortic aneurysms.

Table 5. The comparison of the patients according to COVID-19 positivity

\begin{tabular}{|c|c|c|c|c|c|c|c|c|}
\hline & \multicolumn{3}{|c|}{ COVID-19 (-) } & \multicolumn{3}{|c|}{ COVID-19 (+) } & \multirow[b]{2}{*}{ Test statistics } & \multirow[b]{2}{*}{$p$} \\
\hline & Mean $\pm S D$ & Median & Min-Max & Mean $\pm S D$ & Median & Min-Max & & \\
\hline Age (year) & $74.5 \pm 6.5$ & 76.00 & $60.0-83.00$ & $82.7 \pm 5.5$ & 82.50 & $75.00-90.00$ & $-2.74 \dagger$ & 0.013 \\
\hline Abdominal aortic diameter $(\mathrm{mm})$ & $72.0 \pm 2.3$ & 71.50 & $68.5-74.90$ & $82.0 \pm 5.1$ & 83.95 & $72.30-85.60$ & $89.000 \%$ & 0.001 \\
\hline Aortic neck diameter (mm) & $26.5 \pm 2.9$ & 26.50 & $21.5-31.20$ & $26.8 \pm 5.4$ & 27.85 & $16.50-31.10$ & $-0.131 \dagger$ & 0.897 \\
\hline Aortic neck length (mm) & $17.7 \pm 2.6$ & 17.00 & $14.0-22.00$ & $20.5 \pm 2.4$ & 20.50 & $17.00-24.00$ & $-2.332 \uparrow$ & 0.030 \\
\hline Right iliac diameter (mm) & $23.7 \pm 11.3$ & 21.45 & $14.6-64.50$ & $26.6 \pm 10.3$ & 23.10 & $16.30-44.50$ & $61.500 \ddagger$ & 0.329 \\
\hline Left iliac diameter (mm) & $20.4 \pm 4.4$ & 18.55 & $15.3-31.50$ & $20.9 \pm 4.5$ & 22.45 & $14.30-26.30$ & $-0.231 \dagger$ & 0.819 \\
\hline Procedural duration (min) & $51.2 \pm 6.8$ & 50.00 & $40.0-65.00$ & $54.2 \pm 8.8$ & 55.00 & $40.00-65.00$ & $-0.842 \dagger$ & 0.409 \\
\hline Length of hospital stay (days) & $1.6 \pm 0.7$ & 1.00 & $1.0-3.00$ & $2.3 \pm 0.5$ & 2.00 & $2.00-3.00$ & $76.000 \%$ & 0.040 \\
\hline
\end{tabular}

SD: Standard deviation; Min: Minimum; Max: Maximum; $\uparrow$ Independent samples t-test; $\ddagger$ Mann-Whitney U test.

without COVID-19 (2.3 \pm 0.5 days vs. $1.6 \pm 0.7$ days, respectively; $\mathrm{p}=0.040)$. As the COVID-19-positive patients were asymptomatic, all were discharged to home for self-isolation and home quarantine at the discretion of the treating department. The comparison of the patients with and without COVID-19 is shown in Table 5.

All patients received anticoagulants after the procedure. The COVID-19-negative patients received postoperative acetylsalicylic acid and clopidogrel, while infected patients were given low-molecular-weight heparin (LMWH) for 14 days to prevent thrombotic events (by adjusting the dose appropriate for the weight of the patients), followed by acetylsalicylic acid and clopidogrel. At one month of follow-up, repeated CTA revealed no stent migration or thrombotic events and no mortality was observed during the first 30-day of follow-up.

\section{DISCUSSION}

The COVID-19 pandemic has placed a tremendous strain on healthcare services in Turkey, as in the whole globe. The rapid spread of the pandemic has crippled the treatment of pathologies other than the 
COVID-19 and led to seeking ways for standardquality treatments for all patients. The pandemic has posed certain challenges for high-risk and/or life-threatening medical conditions, if left untreated and AAAs are one of the most fatal aneurysms, unless treated in a timely manner. ${ }^{[6]}$

Before the pandemic era, treatment recommendations are available for AAAs of $>55 \mathrm{~mm}$. Even in case of AAAs of $>60 \mathrm{~cm}$ in size, the risk of complications and rupture has been shown to increase. ${ }^{[7]}$ However, during the pandemic, the American College of Surgeons recommended that surgery should be postponed for abdominal aortic aneurysms (AAAs) larger than $6.5 \mathrm{~cm}$ (Tier 2b) and smaller than $6.5 \mathrm{~cm}$ (Tier 1). ${ }^{[4]}$ The main goal of these guidelines is to decrease the burden of the pandemic on the healthcare services, particularly on the ICU settings, and to prevent occupation of ICU and hospital beds. On the other hand, these recommendations pose certain risks. Although the decision of postponement or cancellation of surgery in patients with an AAA of $>65 \mathrm{~mm}$ is at the discretion of the treating surgeon, patients may experience disappointment and feel themselves desperate. As a result, the question of whether postponing treatment based on the aneurysmal diameter alone is the right decision or whether other variables should be also considered (i.e., age of the patient and treatment method) is still waiting to be answered.

In a recent study, the absolute risk of mortality increased by 5.4 to $7.7 \%$ in patients with an AAA of $>7 \mathrm{~cm}$ in whom treatment was postponed for three months. ${ }^{[6]}$ In younger patients aged between 60 and 70 years with an AAA of 6 to $6.9 \mathrm{~cm}$, the mortality risk increased by 1.5 to $1.9 \%$, while there was an increase in the mortality risk by 0.2 to $0.8 \%$ in patients aged 60 years with an AAA of 5 to $5.9 \mathrm{~cm}$. The postponement of treatment for six months resulted in an increase in the mortality risk by 2.4 to $4.5 \%$ in patients with an AAA of 6 to $6.9 \mathrm{~cm}$. This study, however, seems not to strictly comply with the recommendations issued during the pandemic.

Endovascular aneurysm repair has become the treatment of choice for AAAs worldwide with a lower perioperative risk and shorter postoperative length of hospital stay. ${ }^{[8]}$ This treatment modality has been used for the treatment of AAAs in $67.2 \%$ patients in the United Kingdom, 72.6\% patients in Germany (72.6\%), $75.4 \%$ patients in the United States. ${ }^{[9]}$

To utilize healthcare services effectively, treatment of AAAs in elderly patients requiring open surgical repair should be first postponed during the pandemic, since the need for ICU stay and a higher amount of healthcare resources in this patient population. ${ }^{[10-12]}$ The rate of ICU need is as low as $1 \%$ in patients undergoing elective EVAR and requires minimum utilization of healthcare resources such as personnel, mask, and gown. ${ }^{[13]}$ In our study, the mean length of hospital stay was $1.8 \pm 0.8$ (range, 1 to 3 ) days, consistent with the literature.

A growing number of evidence has shown that aortic inflammation plays a key role in the progression of the existing aneurysm and, as a result, some authors have proposed that COVID-19 infection may worsen the aortic aneurysm, leading to increased severity of complications. ${ }^{[14]}$ If anatomically feasible, large aneurysms can be treated with EVAR without any delay in patients with COVID-19 infection, since these patients are more prone to postoperative complications following open surgery due to existing lymphopenia indicating immunosuppression. ${ }^{[15]}$

During the most virulent period of the outbreak, several studies have reported that EVAR is the first-line treatment for AAAs and it decreases the workload of surgeons. ${ }^{[6,16-18]}$ In our study, we also avoided open surgical repair due to the limited capacity of mechanical ventilators and ICU beds in our facility and performed EVAR for AAAs of $\geq 6.5 \mathrm{~cm}$ under spinal anesthesia to minimize aerosol production related to intubation, particularly in patients with COVID-19 infection. Many studies have shown that intubation is a definite aerosolgenerating procedure which potentially endangers the health of the healthcare workers. ${ }^{[19]}$ In addition, open surgery requires a number of staff in the operating room, while EVAR can be performed in the angiography lab with a minimum number of staff. Similarly, we performed all EVAR procedures with only a surgeon, a nurse, and a CTA technician in the angiography lab, ensuring cost-saving and minimizing transmission of infection. ${ }^{[20]}$

Although the outcomes of endograft implantation are still unclear in COVID-19 patients, there are some reports showing a possible link between COVID-19 infection and arterial and venous thrombotic complications. In the light of these data, systemic heparinization with an ACT of $\geq 300 \mathrm{sec}$ is recommended for COVID-19 patients undergoing EVAR. ${ }^{[17,21-22]}$ In accordance with the recommendations, we also administered systemic heparinization with an ACT of $>300 \mathrm{sec}$ to COVID-19-positive patients to prevent thrombotic 
events and none of the patients had any thrombotic complications in this study.

Furthermore, several reports have indicated that limb thrombosis risk should be taken into consideration, as COVID-19 patients may have evidence of a prothrombotic state, and anticoagulation is recommended for these patients. ${ }^{[23,24]}$ Similarly, in our study, we applied LMWH to six COVID-19 patients for 14 days to prevent thrombotic events, followed by acetylsalicylic acid and clopidogrel. More importantly, visitors and caregivers were not allowed in the hospital setting during the pandemic which may pose adverse psychological effects on the mental health of patients undergoing open surgical repair due to the lack of care and sympathy from their loved ones. However, EVAR allows a rapid discharge to home without such adverse consequences.

The postponement or cancellation of AAA treatment in accordance with the recent guideline recommendations during the pandemic not only increases the mortality risk, but also result in several concerns. The accumulation of postponed surgeries would put a serious strain with the increased workload on both the healthcare facilities and healthcare workers including operating rooms and ICUs. After the end of the pandemic, the possible increase in the number of patients in whom treatment has postponed or canceled is expected to reduce the capacity and resources of healthcare facilities. ${ }^{[25]}$

Nonetheless, there are some limitations to this study. The low number of cases and patients with COVID-19 and the low duration of follow-up are the limitations of our study.

In conclusion, COVID-19 has put a halt to almost all healthcare services and workers worldwide and many has a struggle against this devastating pandemic. While being at the core of the pandemic, surgeons should adopt the concept of "do the right thing, the right way, for the right reasons". The operation can be delayed in patients requiring open surgical repair or in those with small AAAs. Even in patients with COVID-19 having an AAA of $>6.5 \mathrm{~cm}$, we believe that EVAR can be used as the first choice of treatment and be performed successfully in the angiography lab with a minimum number of staff and minimum utilization of healthcare resources and without the need for intubation or ICU stay. Additional anticoagulation is recommended to prevent postoperative limb or graft thrombosis.
However, further large-scale, prospective studies are needed to draw a firm conclusion.

\section{Declaration of conflicting interests}

The authors declared no conflicts of interest with respect to the authorship and/or publication of this article.

\section{Funding}

The authors received no financial support for the research and/or authorship of this article.

\section{REFERENCES}

1. Guan WJ, Ni ZY, Hu Y, Liang WH, Ou CQ, He JX, et al. Clinical characteristics of coronavirus disease 2019 in China. N Engl J Med 2020;382:1708-20.

2. Verikokos C, Lazaris AM, Geroulakos G. Doing the right thing for the right reason when treating ruptured abdominal aortic aneurysms in the COVID-19 era. J Vasc Surg 2020;72:373-4.

3. Ng JJ, Ho P, Dharmaraj RB, Wong JCL, Choong AMTL. The global impact of COVID-19 on vascular surgical services. J Vasc Surg 2020;71:2182-3.e1.

4. ACS. 2020. (COVID-19: elective case triage guidelines for surgical care). Available at: https://www.facs.org/ covid-19/clinical-guidance/elective-case/vascular-surgery. [Accessed: May 3, 2020].

5. Shin DW, Cho J, Kim SY, Guallar E, Hwang SS, Cho $\mathrm{B}$, et al. Delay to curative surgery greater than 12 weeks is associated with increased mortality in patients with colorectal and breast cancer but not lung or thyroid cancer. Ann Surg Oncol 2013;20:2468-76.

6. McGuinness B, Troncone M, James LP, Bisch SP, Iyer V. Reassessing the operative threshold for abdominal aortic aneurysm repair in the context of COVID-19. J Vasc Surg 2021;73:780-8.

7. Günertem E, Akay T, Sargın M, Erdil N, Uğuz E, Aslım $\mathrm{E}$, et al. Paradigm shifts in terms of vascular emergencies during COVID-19 outbreak: An expert opinion report. Turk J Vasc Surg 2020;29:208-21

8. Chaikof EL, Dalman RL, Eskandari MK, Jackson BM, Lee WA, Mansour MA, et al. The Society for Vascular Surgery practice guidelines on the care of patients with an abdominal aortic aneurysm. J Vasc Surg 2018;67:277.e2.

9. Trenner M, Salvermoser M, Reutersberg B, Busch A, Schmid $\mathrm{V}$, Eckstein $\mathrm{HH}$, et al. Regional variation in endovascular treatment rate and in-hospital mortality of abdominal aortic aneurysms in Germany. Vasa 2020;49:107-14.

10. Lederle FA, Freischlag JA, Kyriakides TC, Padberg FT Jr, Matsumura JS, Kohler TR, et al. Outcomes following endovascular vs open repair of abdominal aortic aneurysm: A randomized trial. JAMA 2009;302:1535-42.

11. Matsumura JS, Stroupe KT, Lederle FA, Kyriakides TC, Ge L, Freischlag JA; Open Versus Endovascular Repair (OVER) Veterans Affairs Cooperative Study Group. Costs of repair of abdominal aortic aneurysm with different devices in a multicenter randomized trial. J Vasc Surg 2015;61:59-65. 
12. Jetty $P$, Hebert $P$, van Walraven C. Long-term outcomes and resource utilization of endovascular versus open repair of abdominal aortic aneurysms in Ontario. J Vasc Surg 2010;51:577-83,.

13. Rajasinghe HA, Miller LE, Krajcer Z. Early outcomes with fast-track EVAR in teaching and nonteaching hospitals. Ann Vasc Surg 2018;49:134-43.

14. MA3RS Study Investigators. Aortic wall inflammation predicts abdominal aortic aneurysm expansion, rupture, and need for surgical repair. Circulation 2017;136:787-97.

15. Qin C, Zhou L, Hu Z, Zhang S, Yang S, Tao Y, et al. Dysregulation of immune response in patients with coronavirus 2019 (COVID-19) in Wuhan, China. Clin Infect Dis 2020;71:762-8.

16. Shih M, Swearingen B, Rhee R. Ruptured abdominal aortic aneurysm treated with endovascular repair in a patient with active COVID-19 infection during the pandemic. Ann Vasc Surg 2020;66:14-7.

17. Yammine H, Ballast JK, Poulsen N, Arko FR 3rd. Endovascular aortic repair in an asymptomatic COVID19-positive patient with a symptomatic inflammatory abdominal aortic aneurysm. J Vasc Surg Cases Innov Tech 2020;6:531-3.

18. Rinaldi LF, Marazzi G, Marone EM. Endovascular treatment of a ruptured pararenal abdominal aortic aneurysm in a patient with coronavirus disease-2019: Suggestions and case report. Ann Vasc Surg 2020;66:18-23.
19. Tran K, Cimon K, Severn M, Pessoa-Silva CL, Conly J. Aerosol generating procedures and risk of transmission of acute respiratory infections to healthcare workers: A systematic review. PLoS One 2012;7:e35797.

20. Giacomelli E, Dorigo W, Fargion A, Calugi G, Cianchi G, Pratesi C. Acute thrombosis of an aortic prosthetic graft in a patient with severe COVID-19-related pneumonia. Ann Vasc Surg 2020;66:8-10.

21. Marone EM, Rinaldi LF. Upsurge of deep venous thrombosis in patients affected by COVID-19: Preliminary data and possible explanations. J Vasc Surg Venous Lymphat Disord 2020;8:694-5.

22. Zhai Z, Li C, Chen Y, Gerotziafas G, Zhang Z, Wan J, et al. Prevention and treatment of venous thromboembolism associated with coronavirus disease 2019 infection: A consensus statement before guidelines. Thromb Haemost 2020;120:937-48.

23. Klok FA, Kruip MJHA, van der Meer NJM, Arbous MS, Gommers DAMPJ, Kant KM, et al. Incidence of thrombotic complications in critically ill ICU patients with COVID-19. Thromb Res 2020;191:145-7.

24. Leong Tan GW, Chandrasekar S, Lo ZJ, Hong Q, Yong E, Lingam P, et al. Early experience in the COVID-19 pandemic from a vascular surgery unit in a Singapore tertiary hospital. J Vasc Surg 2020;72:12-5.

25. Grabowski DC, Joynt Maddox KE. Postacute care preparedness for COVID-19: Thinking ahead. JAMA 2020;323:2007-8. 